\title{
Intervenções fundamentais em cirurgia: diérese, hemostasia e síntese
}

\section{Fundamental interventions in surgery: dieresis, hemostasis and synthesis}

\author{
Aldo Cunha Medeiros ${ }^{1}$, Antônio Medeiros Dantas Filho²
}

1. Professor Titular de Técnica Operatória, Departamento de Cirurgia, Coordenador do Núcleo de Cirurgia Experimental, Universidade Federal do Rio Grande do Norte (UFRN), Natal-RN, Brasil.

2. Professor Adjunto, Departamento de Cirurgia, UFRN, Natal-RN, Brasil.

Revisão realizada no Departamento de Cirurgia, UFRN, Natal, Brazil.

Suporte financeiro: nenhum.

Conflito de interesse: nenhum.

Endereço para correspondência: Departamento de Cirurgia, UFRN, Av. Nilo Peçanha 620, Natal, RN, Brasil. E-mail: cirurgex.ufrn@gmail.com

Submetido: Setembro 16, 2018. Aceito após revisão: Outubro 02, 2018.

\section{ABSTRACT}

Objective: This article aims to review and describe relevant aspects and recent advances in dieresis, hemostasis and synthesis, the fundamental times of surgery. Methods: Surgery has evolved over centuries, but the concepts of dieresis, hemostasis and synthesis continue, with many updates. The present review was carried out from bibliographic research in the electronic databases Pubmed and SciElo, in addition to consulting specialized books on operative technique. Results: In the past, large incisions extending along the abdomen, chest and limbs were the standard approach to access the organs and tissues of these anatomical regions. However, with advances in technology and improvements in surgical technique, surgery has progressed to minimize trauma to the patient while maintaining surgical principles. In this review it was demonstrated that no matter the length of the incisions and the size of trauma. The principles of good technique that always include dieresis, hemostasis and synthesis continue the same, and this was described in the present work. Conclusion: Whatever the organ or anatomical region to be operated on, The concepts described in this paper reaffirm that the surgeon can't deviate from the precepts of good surgical practice, always attending to the prerequisites of a good incision, perfect hemostasis and impeccable synthesis of tissues.

Keywords: Surgery. Surgical incisions. Hemostasis. Synthesis. 


\section{RESUMO}

Objetivo: Este artigo tem o objetivo de revisar e descrever aspectos relevantes e recentes avanços sobre diérese, hemostasia e síntese, os tempos fundamentais da cirurgia. Métodos: A cirurgia evoluiu ao longo de séculos, mas os conceitos a respeitos de diérese, hemostasia e síntese continuam, com muitas atualizações. A presente revisão foi realizada a partir de pesquisa bibliográfica nas bases de dados eletrônicas Pubmed e SciElo, além de consulta a livros especializados em técnica operatória. Resultados: No passado, grandes incisões que se estendiam ao longo do abdome, tórax e membros, eram a abordagem padrão para acessar os órgãos e tecidos dessas regiões anatômicas. No entanto, com os avanços da tecnologia e as melhorias na técnica cirúrgica, a cirurgia progrediu para minimizar o trauma ao paciente, mantendo os princípios cirúrgicos. Entretanto, nesta revisão ficou demonstrado que não importa a extensão das incisões e o porte do trauma. Os princípios da boa técnica que quase sempre incluem diérese, hemostasia e síntese continuam os mesmos, e é o que ficou descrito no presente trabalho. Conclusão: Seja qual for o órgão ou a região anatômica a ser operada, os conceitos aqui descritos reafirmam que não há como o cirurgião fugir dos preceitos da boa prática cirúrgica, sempre atendo aos pré-requisitos de uma boa incisão, perfeita hemostasia e impecável síntese dos tecidos.

Descritores: Técnica. Cirurgia. Incisão cirúrgica. Hemostasia. Síntese.

\section{INTRODUCTION}

Intervenção cirúrgica é o conjunto de gestos manuais, auxiliados por instrumentos, que o cirurgião realiza para a execução de ato operatório com finalidade terapêutica, estética ou diagnóstica. As intervenções fundamentais, também chamadas tempos fundamentais da cirurgia, são atos cirúrgicos considerados simples, comuns à maioria das operações, que praticados em sequência, permitem a realização de operações complexas. São diérese, hemostasia e síntese.

\section{DIÉRESE}

Diérese é uma expressão derivada de palavras do Latim (diaerese) e do Grego (diairesis) ambas significando divisão, incisão, secção, separação, divulsão. Pode ser definida como o ato do cirurgião provocar uma solução de continuidade nos tecidos, ou uma via de acesso a cavidades anatômicas, órgãos e tecidos. 
Intervenções fundamentais em cirurgia: diérese, hemostasia e síntese Medeiros AC, Dantas-Filho AM

\section{Tipos de diérese}

1. Incisão - é a manobra que secciona ou incisa os tecidos. É praticada com instrumentos cortantes que seccionam os tecidos moles, mais frequentemente por meio de lâmina de bisturi, produzindo ferida incisa. Considera-se que o melhor estímulo para uma boa cicatrização é o corte frio e decisivo com lâmina de bisturi frio e afiado. Incisões podem ser praticadas com outros meios cortantes como eletrocautério, laser e ultrassom. Esses meios devem ser usados criteriosamente, evitando-se lesões e reações locais indesejáveis.

\section{Incisões praticadas com bisturi eletro-eletrônico (diatermia) e ultrassom:}

A diatermia tem sido amplamente utilizada em todos os tipos de operações tanto para a diérese quanto para a hemostasia. A experiência cirúrgica é longa com bisturi elétrico/eletrônico, mas continua controverso se a diatermia para a diérese prejudica ou não o processo de cicatrização ${ }^{1}$. Alguns estudos com avaliação da diatermia podem ser citados. Ela pode provocar maior inflamação e necrose de tecidos do que o bisturi frio e pode induzir a formação de aderências, nos casos de cirurgia abdominal2,3. Redução significativa da resistência à tensão das feridas cirúrgicas suturadas, bem como aumento no tempo de cicatrização com diatermia tem sido citados ${ }^{3}$. Um estudo randomizado mostrou que o uso de bisturi frio ou a diatermia são equivalentes, pois a reação inflamatória ao abrir o abdome com diatermia seria menos importante, pois o eletrocautério promove a diérese e a homeostasia ao mesmo tempo, reduzindo o tempo operatório e o sangramento ${ }^{4}$. Estudo comparando eletrocautério e incisão com bisturi frio demonstrou que são equivalentes quanto à avaliação histológica e conteúdo de colágeno nas feridas 5 .

Estudo de metanálise concluiu que, comparada ao eletrocautério, a dissecção e incisão com bisturi ultrassônico (harmônico) apresenta vantagens significativas na diminuição da necessidade de drenagem pós-operatória, desenvolvimento de seroma, perda sangüínea intra-operatória e complicações de feridas na mastectomia radical para câncer de mama, sem aumentar o tempo cirúrgico ${ }^{6}$. Na videocirurgia e na cirurgia hepática o bisturi ultrassônico tem sido usado com muito sucesso. 
Intervenções fundamentais em cirurgia: diérese, hemostasia e síntese

Medeiros AC, Dantas-Filho AM

Todos os meios e equipamentos são úteis em cada caso, restando ao cirurgião usá-los criteriosamente. Quando bem utilizados, obedecendo aos princípios do trauma mínimo e com bons conhecimentos de anatomia e técnica operatória, todos os instrumentos e técnicas de diérese são bons.

\section{Incisões com raios laser}

O termo LASER é uma sigla derivada do Inglês, Light amplification by stimulated emission of radiation. O laser é composto de ondas luminosas, em raios infravermelhos concentrados e de alta potência. Os raios têm o mesmo comprimento de onda, mesma cor e vibram na mesma frequência. A luz á ordenada, correspondendo a um feixe de luz em que todos os fótons são idênticos. Repercussões biológicas das incisões com raios laser: os tecidos são vaporizados; o tecido vizinho não é afetado; os vasos sanguíneos e linfáticos são laqueados por fotocoagulação; a cicatrização não é significativamente prejudicada; os microrganismos são eliminados localmente; a dor no pós-operatório é menor do que com outras técnicas de diérese; e o tempo operatório é menor ${ }^{7-9}$.

\section{Qualidades de uma boa incisão:}

1. A incisão deve ter tamanho adequado de modo que permita acesso fácil aos órgãos a serem abordados e boa visibilidade do campo operatório.

2. A incisão deve ter bordas regulares.

3. Obedecer às linhas de força da pele da região da intervenção cirúrgica (Linhas de Kraissl, Linhas de Langer).

4. Atravessar os tecidos respeitando a anatomia regional, incisando um plano de cada vez.

5. Não comprometer vasos e nervos importantes da região operada ${ }^{10}$.

2. Divulsão - neste tipo de diérese o cirurgião realiza o afastamento dos tecidos sem seccioná-los, aproveitando o plano de clivagem ou sua constituição fasciculada, como por exemplo o tecido muscular. Ela pode ser feita com tesoura de pontas rombas, pinças hemostáticas, pinça de Mixter, afastador de Farabeuf, tentacânula e outros instrumentos (Figura 1). 

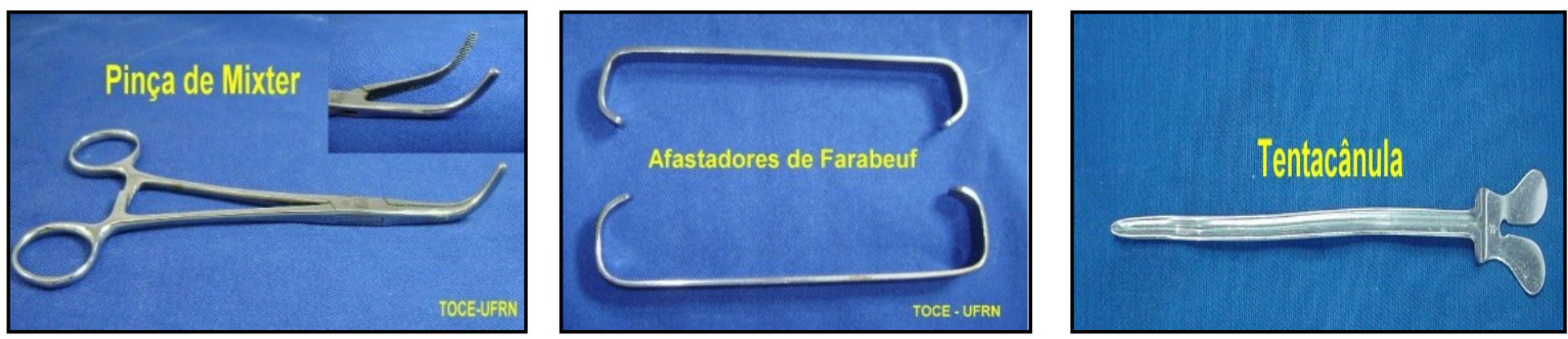

Figura 1 - Pinça de Mixter, afastador de Farabeuf, tentacânula

3. Punção - é o tipo mais simples de diérese, realizado com agulha ou com trocáter que penetra nos tecidos e paredes que dão acesso a cavidades, separando-os sem seccioná-los. Tem várias finalidades, como dar acesso à cavidade abdominal durante a cirurgia laparoscópica, drenagem de coleções líquidas de cavidades e órgãos, coleta de fragmentos de órgãos e tecidos para exames diagnósticos, injeção de contrastes e medicamentos, etc.

4. Descolamento - é realizado pela separação romba dos tecidos através de um espaço anatômico virtual. Como exemplos, pode-se citar o descolamento da vesícula biliar do leito hepático durante a colecistectomia; a manobra de Kocher que consiste no descolamento do duodeno da parede posterior da cavidade abdominal.

5. Dilatação - usada para aumentar o calibre ou o diâmetro de vias naturais estenosadas, canais, ductos e vasos. É obtida pela ruptura de fibras musculares ou de tecido fibroso. Como exemplos, podem ser citados dilatação do colo do útero, dilatação de estenose do esôfago, da uretra, etc.

6. Serração - realizada por meio de serras, mormente na cirurgia ortopédica e cardíaca.

A diérese pode ser auxiliada por técnicas de dissecção mediante emprego de pinças de dissecção, gaze montada em pinças e manobras digitais, com o objetivo de poupar estruturas nobres como nervos e vasos. A diérese praticada de maneira clássica tem sido substituída por vias de acesso minimamente invasivas, com o objetivo de reduzir o dano tecidual, minimizar a resposta endócrina metabólica ao trauma, acelerar a recuperação do paciente e garantir resultados mais estéticos. As incisões são mínimas, 
Intervenções fundamentais em cirurgia: diérese, hemostasia e síntese

Medeiros AC, Dantas-Filho AM

e mediante diérese, dissecção romba, secção cortante ou divulsão, são introduzidos nas

cavidades catéteres percutâneos e trocáteres para videocirurgia.

\section{Instrumentos utilizados na diérese}

Quadro 1 - Tipos de manobra e instrumentos utilizados.

\begin{tabular}{|l|l|}
\hline \multicolumn{1}{|c|}{ MANOBRAS } & \multicolumn{1}{c|}{ INSTRUMENTOS DE DIÉRESE } \\
\hline INCISÃO & Bisturi, serra, tesoura, cisalha, faca, bisturi elétrico, laser. \\
\hline SECÇÃO & Tesoura, serra, lâmina afiada, bisturi elétrico, laser, ultrassom. \\
\hline DIVULSÃO & Tesoura, pinça, afastador, tentacânula. \\
\hline PUNÇÃO & Trocartes, agulhas \\
\hline DILATAÇÃO & Vela de Hegar, Dilatador de Beniqué. \\
\hline SERRAÇÃO & Serra (cirurgia óssea) \\
\hline
\end{tabular}

\section{Bisturis}

O bisturi elétrico utiliza corrente farádica de alta frequência para corte e coagulação por diatermia (Figura 2).

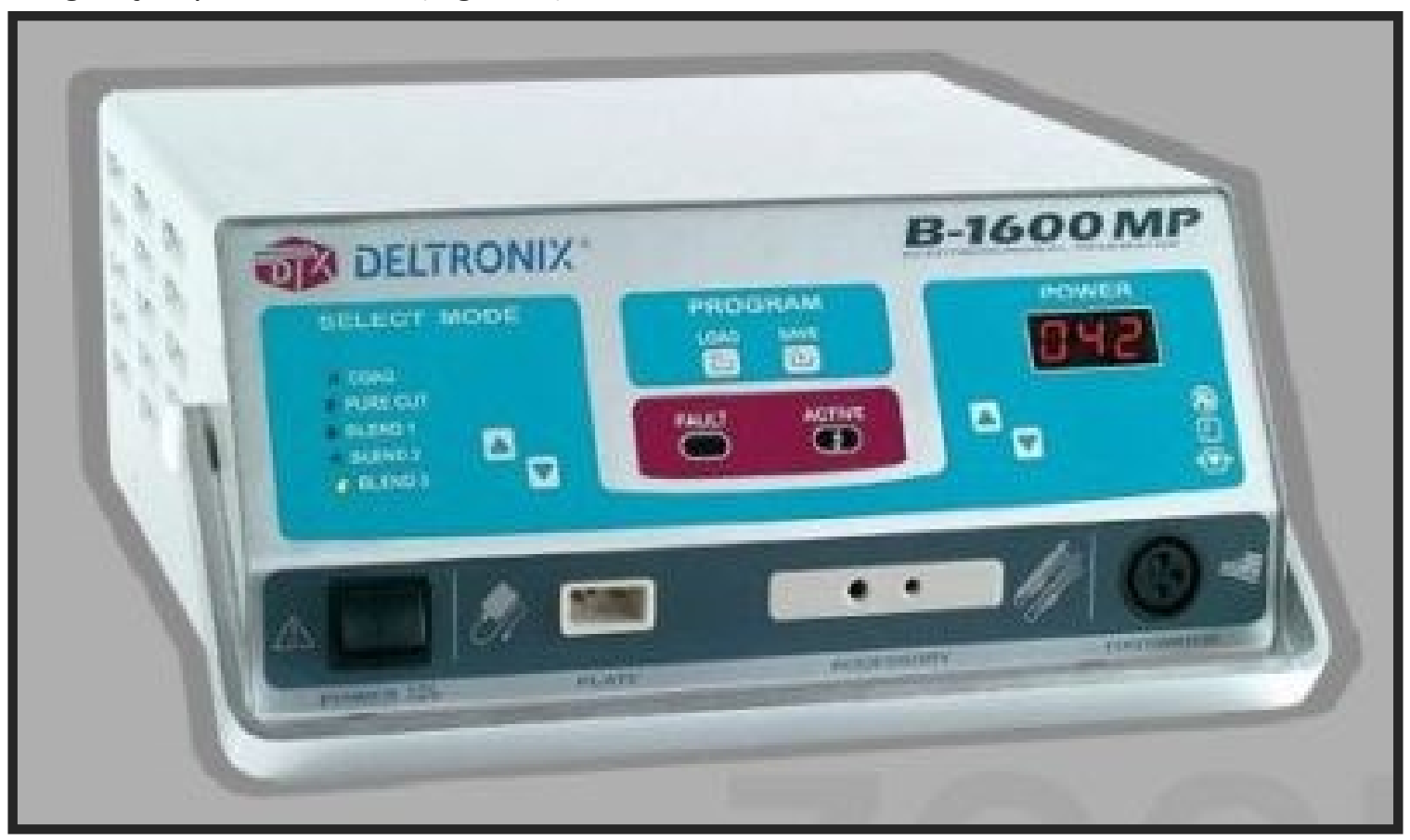

Figura 2 - Bisturi elétrico/eletrônico

O bisturi harmônico/ultrassônico utiliza tecnologia à base de ultrassom para corte e coagulação. (Figura 3). 


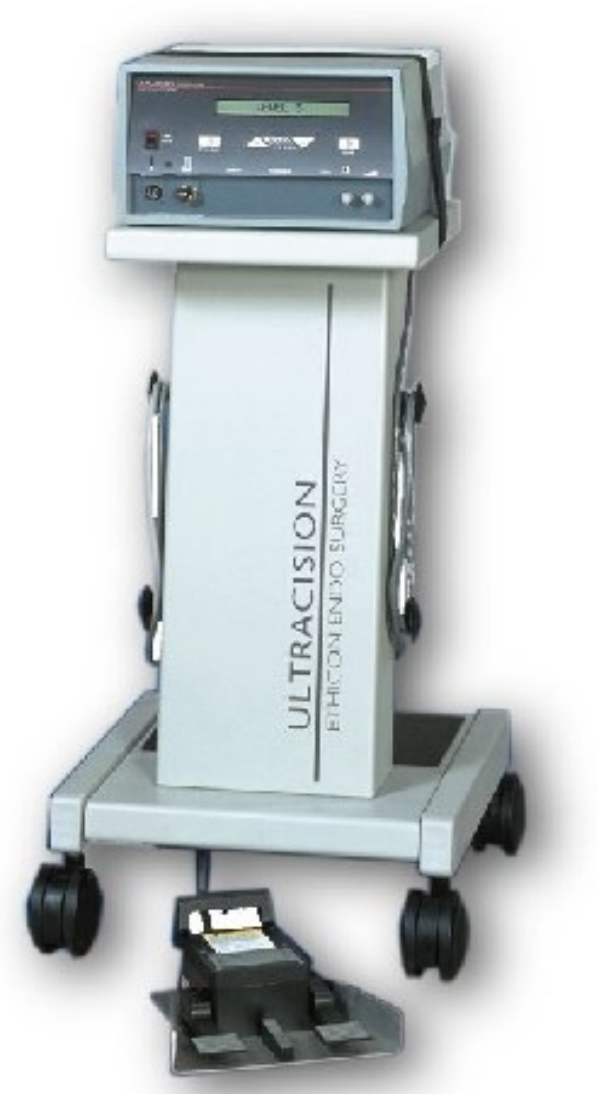

Figura 3 - Bisturi harmônico ${ }^{22}$

Quanto ao bisturi frio, existem vários tamanhos e formas de lâminas de bisturi, todas descartáveis. As lâminas para bisturi podem ser classificadas quanto ao seu formato e aplicabilidade.

Os cabos mais utilizados são: (figura 4)

Cabo no 3 - Permite o encaixe de lâminas pequenas, que possibilitam incisões mais críticas, delicadas (lâminas no 10, 11, 12, 15).

Cabo no 4 - Utiliza lâminas maiores, são mais usados em procedimentos com grandes incisões (lâminas no 20, 21, 22, 23, 24, 25). 


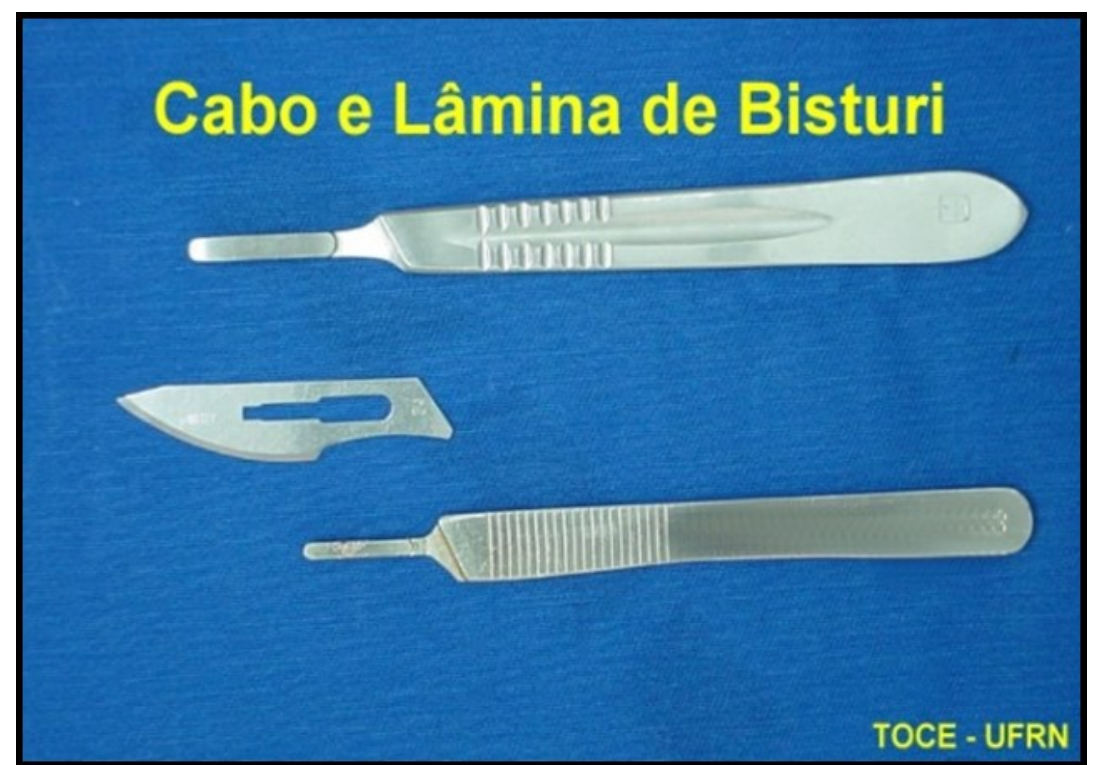

Figura 4 - Cabos de bisturi números 3 e 4, com as respectivas lâminas descartáveis.

\section{Tesouras}

Na rotina cirúrgica são usadas as tesouras de Mayo para fáscias e corte de fios (Figura 5), e as tesouras de Metzenbaum, para a diérese mais delicada de tecidos (Figura 6). Existem de vários tamanhos, retas e curvas, sendo as mais longas utilizadas em cavidades, alcançando estruturas mais profundamente situadas.

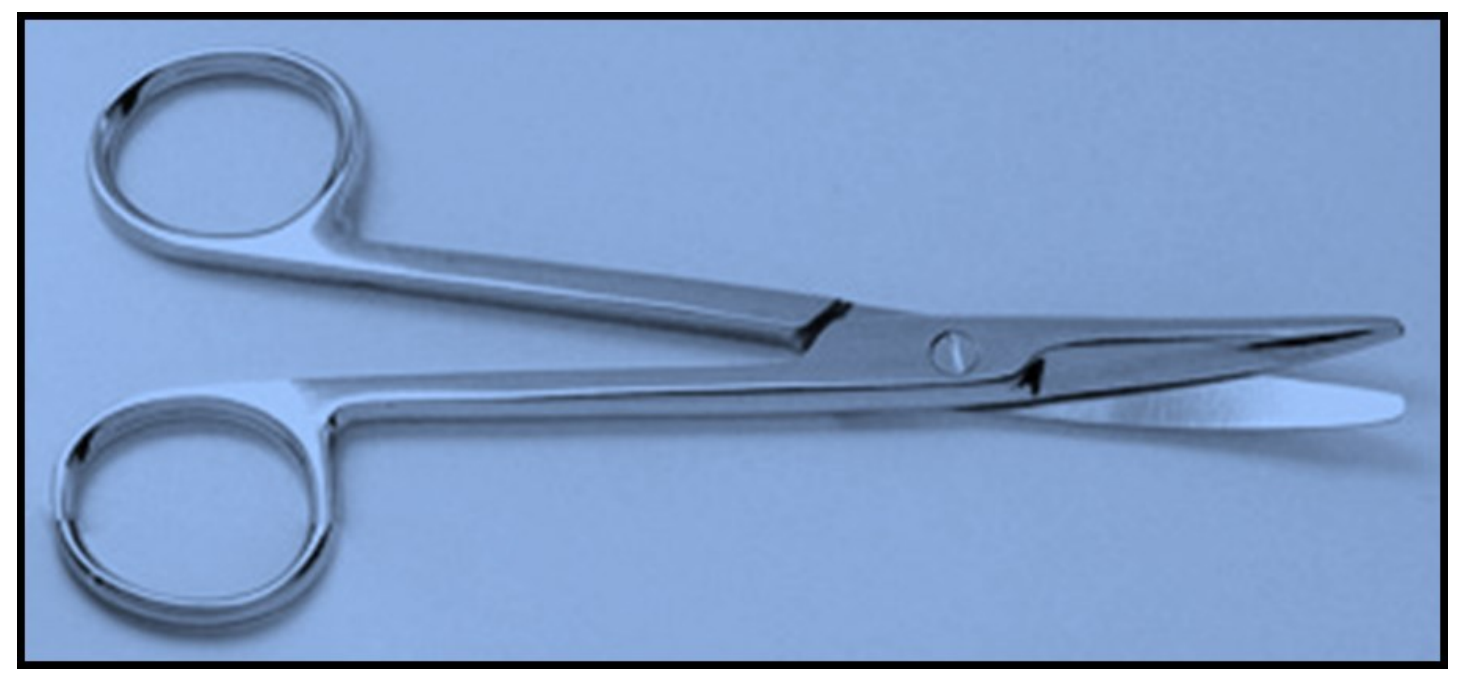

Figura 5 - Tesoura de Mayo. 


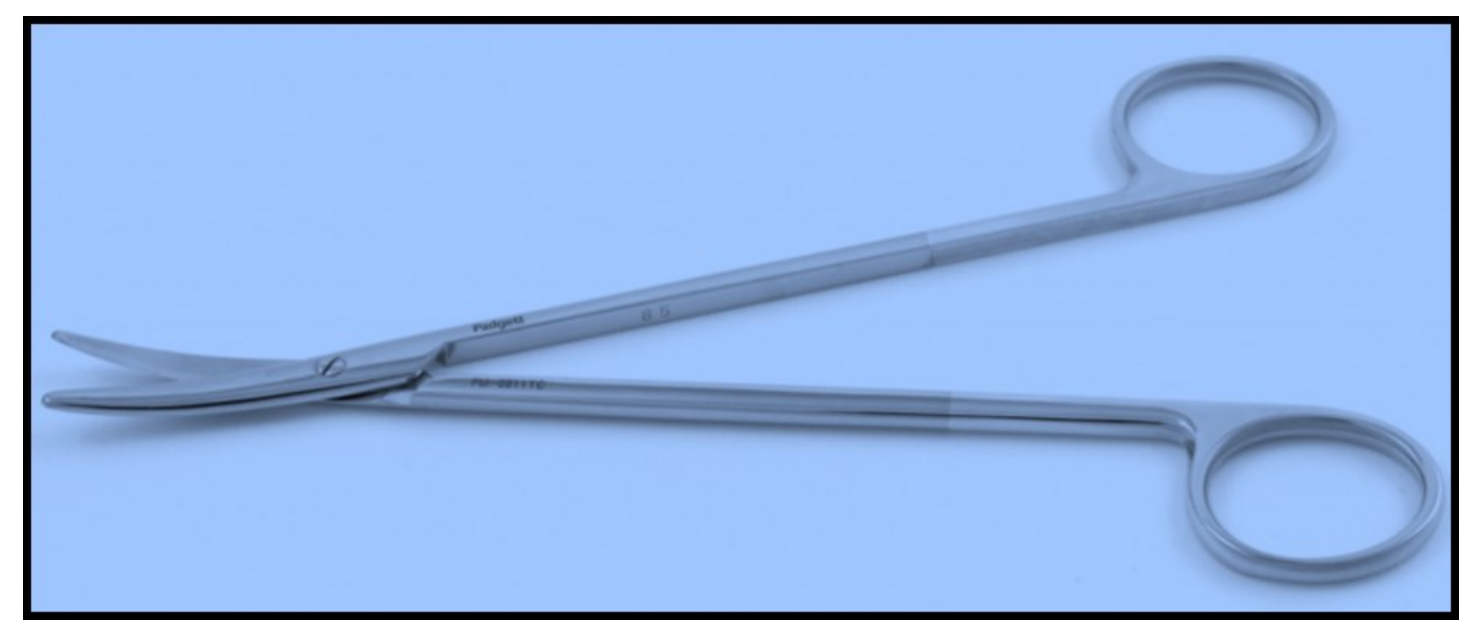

Figura 6 - Tesoura de Metzenbaum.

\section{HEMOSTASIA}

A parada de perdas sanguíneas pode ser espontânea ou fisiológica pela ação dos mecanismos de vasoconstricção, ou cirúrgica (provocada pelo cirurgião). Etimologicamente, hemostasia deriva de hemo=sangue; stasis= parar, deter.

A hemostasia cirúrgica é o conjunto de manobras que tem o objetivo de prevenir, diminuir ou deter o sangramento, garantindo uma boa visibilidade do campo operatório e melhor condição técnica. A falta de êxito nesta etapa do ato operatório pode prejudicar a cicatrização e induzir a infecção.

A hemostasia é um dos aspectos fundamentais que o cirurgião não pode nem deve negligenciar. Em primeiro lugar, para evitar a perda de sangue que pode comprometer a saúde e até a vida do paciente; 2ํㅜ para manter o campo operatório limpo, permitindo reconhecer bem os elementos anatômicos; em terceiro lugar, para o maior êxito operatório, evitando a formação de hematomas e infecção consequente.

No conceito hematológico, hemostasia é o conjunto de mecanismos para manter o sangue fluido entre coagulação e fibrinólise, impedindo a formação de trombos e perdas hemorrágicas. Para manter esse equilíbrio vários fatores colaboram: vascular, plaquetário, fibronólise e fatores da coagulação. Tanto nos pacientes com alterações nos fatores de coagulação quanto nas operações com grandes perdas sanguíneas, pode haver depleção da reserva hemostática do paciente, levando à coagulação intravascular disseminada, complicação gravíssima no paciente cirúrgico. Portanto, a hemostasia pode ser espontânea ou fisiológica e cirúrgica (pelo cirurgião) Além das manobras 
Intervenções fundamentais em cirurgia: diérese, hemostasia e síntese

Medeiros AC, Dantas-Filho AM

cirúrgicas como pinçamento e ligadura, diatermia, uso de laser e bisturi harmônico, a hemostasia pode ser realizada com agentes antifibrinolíticos, adesivos de fibrina e outros $^{11,12}$.

\section{Classificação dos métodos de hemostasia}

São muitos os métodos e manobras realizadas para a hemostasia. Didaticamente, ela pode ser dividida em:

- Hemostasia prévia ou pré-operatória

- Hemostasia temporária

- Hemostasia definitiva

Hemostasia prévia ou pré-operatória: é realizada antes da intervenção cirúrgica. Visa interromper temporariamente o fluxo sanguíneo para o local da incisão, reduzindo a perda sanguínea, proporcionando campo operatório exangue. Mais frequentemente é praticada nos membros e tem o objetivo de prevenir hemorragia. Eventualmente, pode ser usada em casos de atendimento aos acidentados.

Exemplos:

- Compressão em massa de membros - Faixa de Esmarch, garrote de borracha, faixa pneumática, torniquete.

- Ligadura elástica na base de uma extremidade

- Compressão digital de artérias e veias

- Compressão manual da aorta, da veia cava.

O tipo mais empregado de hemostasia prévia é o garroteamento com torniquete pneumático. A compressão elástica de membros pode ser obtida mediante aplicação de longas tiras elásticas de borracha (Faixa de Esmarch), aplicadas de forma helicoidal no sentido das extremidades para as raízes dos membros, muito utilizada na cirurgia ortopédica (Figura 7). 


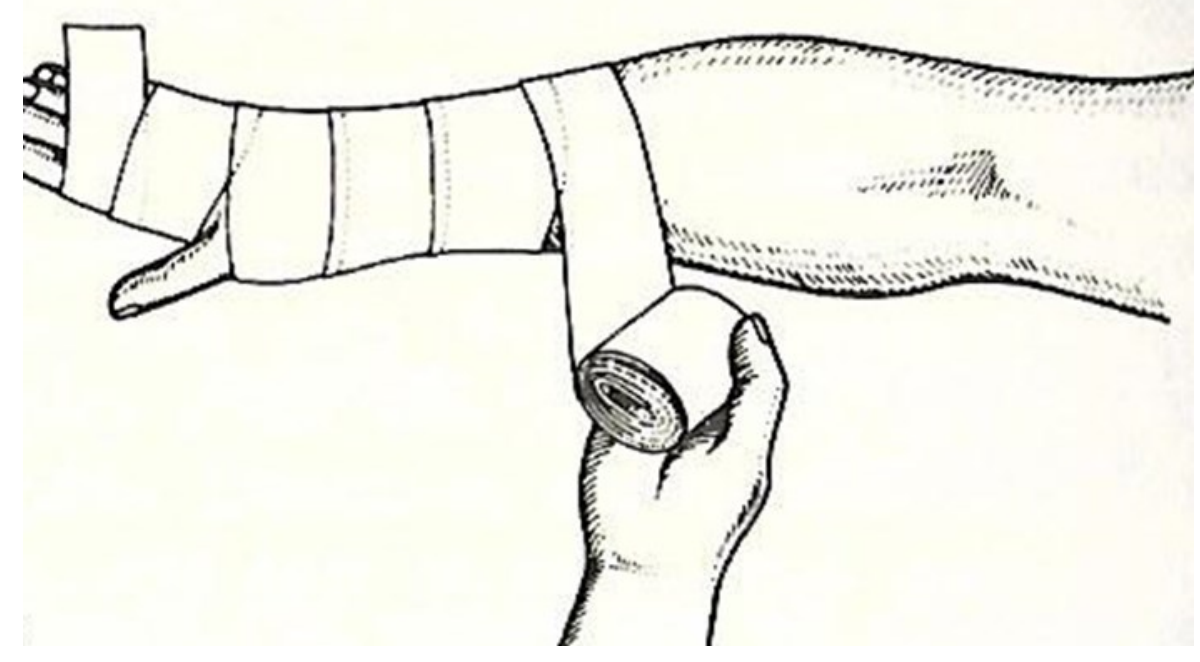

Figura 7 - Faixa de Esmarch. Hemostasia prévia ou pré-operatória ${ }^{10}$.

O protótipo de torniquete pneumático é o manguito do aparelho de pressão que permite a compressão mais uniforme e controlada. Assim, pode ser aplicado isoladamente ou associado ao garroteamento sobre a última volta da faixa de Esmarch, deixando o membro exangue. O manômetro do manguito pode registrar a pressão arterial que deve estar pouco acima da pressão arterial sistólica.

A hemostasia temporária pode ser obtida a partir da compressão vascular mediante emprego de pinças atraumáticas tipo Potts ou Satinsky (Figura 8).

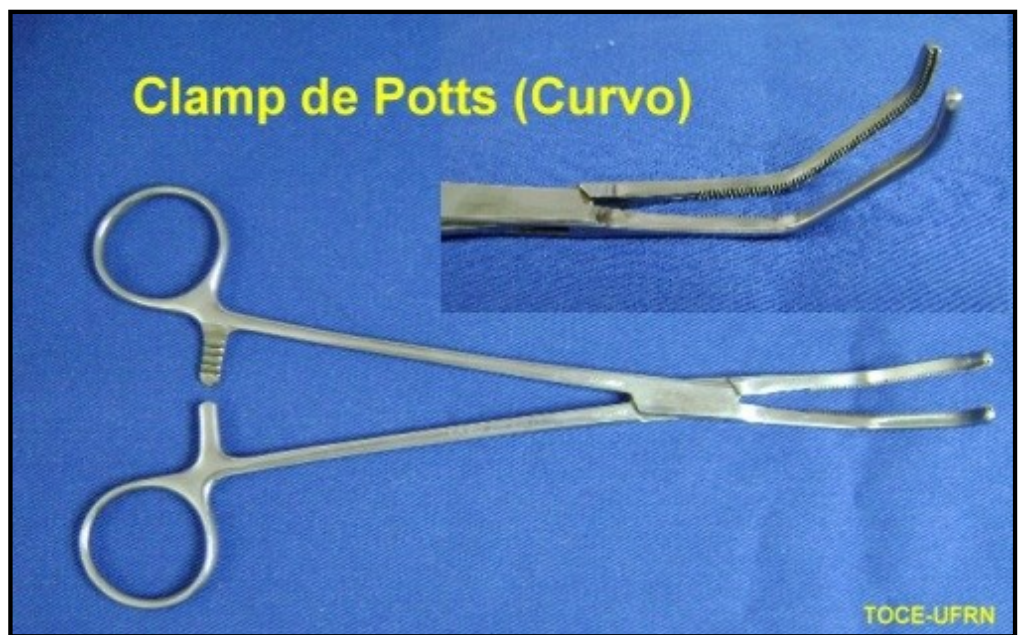




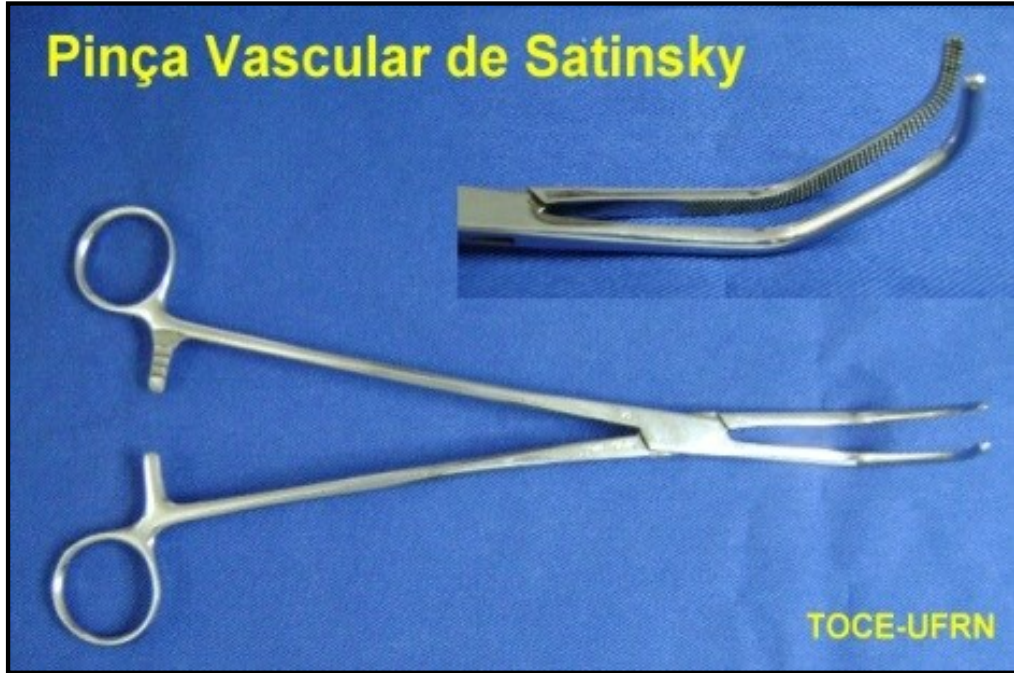

Figura 8 - Pinça de Potts e pinça de Satinsky

A compressão deve ser suave para evitar lesão endotelial e formação de trombos. Outra forma de hemostasia temporária é o tamponamento compressivo realizado mediante colocação de gazes cirúrgicas ou compressas posicionadas no sítio do sangramento de forma manual ou instrumental. O procedimento feito por alguns minutos pode melhorar as condições locais facilitando a hemostasia definitiva, quando necessária.

Em sangramentos do fígado, como no sangramento do trauma hepático, ou durante as hepatectomias, o pinçamento do pedículo hepático no ligamento hepatoduodenal com manobra digital ou com pinça vascular (manobra de Pringle) é a solução de momento, que pode garantir a hemostasia imediata e melhorar as condições para a hemostasia definitiva da lesão hepática. (Figura 9). 


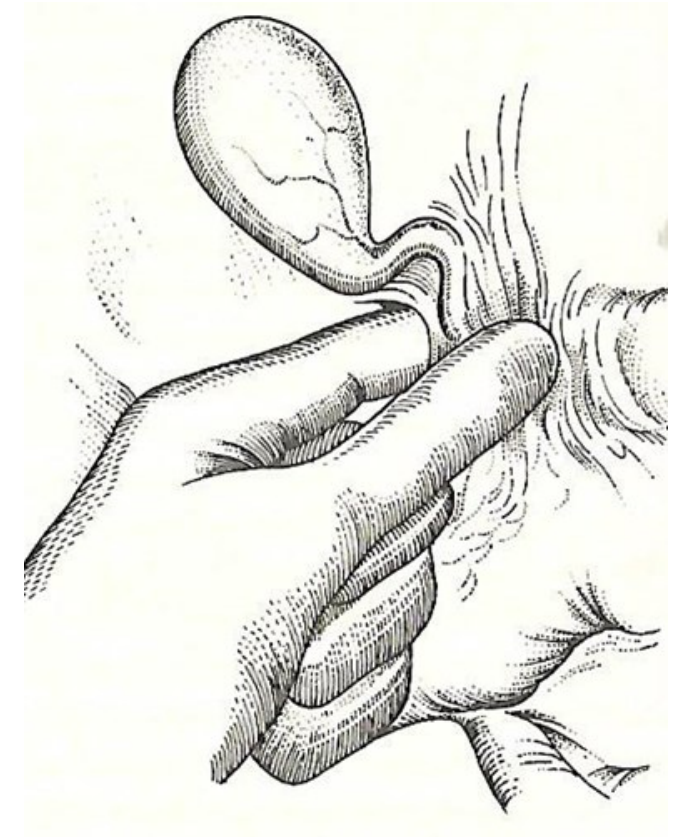

Figura 9 - Manobra de Pringle. Pinçamento digital do ligamento hepatoduodenal, onde se encontram artéria hepática, veia porta e colédoco ${ }^{10}$.

A hemostasia definitiva, é a forma mais frequente e eficiente de hemostasia, é a ligadura do vaso obtida com o isolamento, passagem de dois fios, ligadura à montante e à jusante seguida de secção entre ligaduras. Outras alternativas técnicas consistem do pinçamento do vaso seguido da ligadura, com a utilização de pinças hemostáticas, bem como ligadura com clips metálicos e eletrocoagulação de pequenos vasos.

\section{Instrumental Utilizado na Hemostasia}

A hemostasia definitiva pode ser executada, no decorrer da cirurgia, com o auxílio de instrumentos prensores, dotados de travas ou cremalheiras, denominados pinças hemostáticas. Os vasos são inicialmente pinçados e em seguida ligados ou eletrocoagulados. São diferenciadas, quase sempre, pelo desenho e ranhuras da parte interna dos ramos prensores dos instrumentos. As pinças hemostáticas mais freqüentemente utilizadas na rotina são:

\section{Pinça de Kelly}

Contem ranhuras internas transversais em apenas $2 / 3$ da sua extensão. Os tamanhos variam, havendo as versões reta e curva. (Figura 10) 
Intervenções fundamentais em cirurgia: diérese, hemostasia e síntese Medeiros AC, Dantas-Filho AM

\section{Pinça de Crile}

Em quase tudo é semelhante à pinça de Kelly. A diferença é que tem ranhuras na sua parte prensora em toda sua extensão. Varia de tamanho, nas versões reta e curva. (Figura 10)

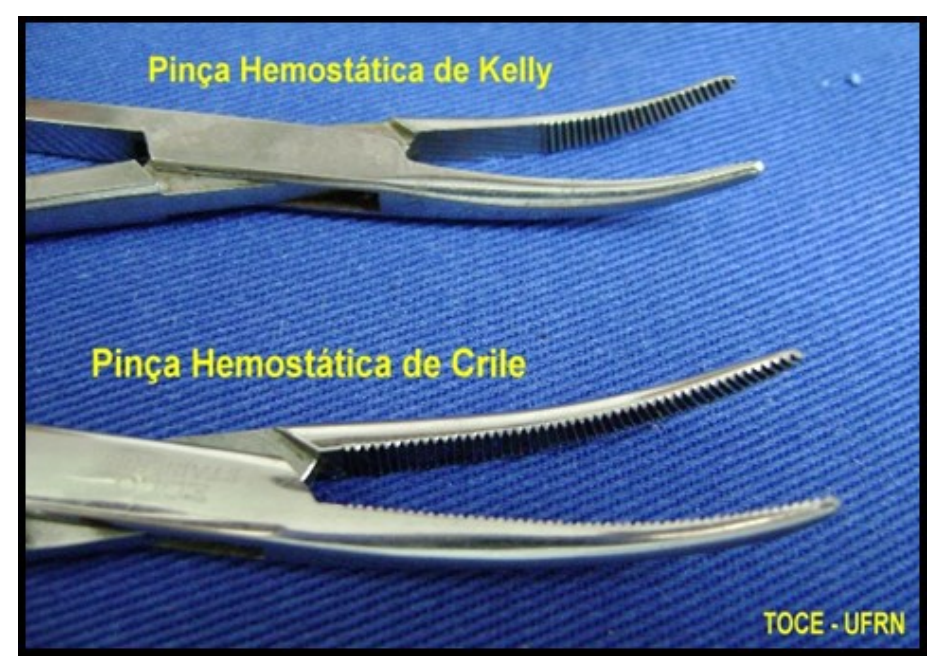

Figura 10 - Piças de Kelly e de Crile

A oclusão de vasos de grosso e médio calibre é obtida de forma mais segura com o emprego de fios e grampos (Clips). Agentes físicos como o calor e a eletrocautério, ou substâncias químicas são empregados na hemostasia de vasos de pequeno calibre. $O$ bisturi elétrico provoca hemostasia pela oclusão dos vasos pelo calor. A passagem da corrente elétrica pela ponta do cautério libera energia térmica que provoca queimadura do tecido, seguida de retração e coagulação dos vasos.

Nos sangramentos difusos de superfícies ósseas ou áreas de grandes descolamentos, a hemostasia pode ser realizada mediante emprego de substâncias químicas ou biológicas como a cera de osso, esponjas de gelatina e adesivos de fibrina.

\section{SÍNTESE}

As complicações mais importantes da feridas são infecção do sítio cirúrgico, deiscência da ferida e hérnia incisional. Evidências experimentais e clínicas sustentam que o desenvolvimento de complicações das feridas operatórias está intimamente relacionado à técnica cirúrgica durante o fechamento das feridas ${ }^{13}$. 
Intervenções fundamentais em cirurgia: diérese, hemostasia e síntese

Medeiros AC, Dantas-Filho AM

Etimologicamente, síntese significa reunir, juntar. É o conjunto de métodos usados para a coaptação ou afrontamento das bordas de uma ferida, traumática ou cirúrgica, com o objetivo de restituir a função e conseguir a cicatrização. A sutura é o método mais frequentemente empregado para a síntese.

\section{Tipos de sutura}

Segundo a permanência:

- Temporária- são suturas que serão retiradas após algum tempo. Como exemplo, suturas de pele.

- Definitiva - suturas que permanecem na intimidade dos tecidos, sendo bons exemplos a sutura do peritônio e da pleura nas intervenções do abdome e tórax respectivamente.

Segundo sua função:

- De coaptação - envolve apenas a coaptação das bordas da ferida (sutura de pele);

- De sustentação - serve para a aproximação das bordas das feridas que tendem a separar-se pela elasticidade dos tecidos;

- De sustentação e coaptação;

- De hemostasia

Segundo a técnica:

- Em pontos separados

- Pontos simples

- Pontos em U

- Pontos em X

- Ponto de Donatti

- Suturas contínuas

- Chuleio simples

- Chuleio ancorado

- Outros tipos de chuleio

Segundo os planos:

- Plano por plano

- Em massa 
Intervenções fundamentais em cirurgia: diérese, hemostasia e síntese

Medeiros AC, Dantas-Filho AM

\section{Segundo a visibilidade}

\section{Estéticas}

- Intradérmicas contínuas ou longitudinais

- Intradérmicas em pontos separados

Suturas externas com pontos aparentes

Na cirurgia convencional aberta, a laparotomia mediana é muito usada, pode ser feita rapidamente e a anatomia da parede abdominal é tal que causa danos mínimos aos músculos, nervos e vasos sanguíneos. Complicações pós-operatórias da ferida, como infecção do sítio cirúrgico, deiscência e hérnia incisional, causam sofrimento ao paciente e geram custos para o sistema de saúde ${ }^{14-16}$. Entre os vários fatores que afetam a taxa de complicações da ferida, os principais podem ser totalmente controlados pelo cirurgião, como a escolha do material de sutura, o método fechamento da ferida e a qualidade das técnicas de sutura.

A síntese é a etapa da operação destinada a reordenar e reaproximar os tecidos. Assim, garante-se a orientação do processo cicatricial e o fornecimento da força tênsil necessária à união tecidual.

A agulha de sutura é o componente mais traumático do material de síntese. As agulhas com fios montados de fábrica provocam mínimo trauma. As agulhas podem ser traumáticas e atraumáticas. As traumáticas, cortantes ou triangulares são utilizadas para síntese da pele ou de outros tecidos resistentes, possuem extremidade triangular e cortante. As agulhas atraumáticas possuem extremidades e corpo cilíndricos e são utilizados para as suturas internas de órgãos e tecidos delicados. As agulhas cilíndricas oferecem maior resistência à passagem pelos tecidos, mas reduzem o trauma tecidual.

Um bom exemplo de sutura a ser executada com boa técnica, por ser muito comum, é a sutura da aponeurose de incisão mediana do abdome, que será aqui utilizada como exemplo de como deve ser realizada a síntese corretamente.

Recomendações sobre como realizar a síntese de uma incisão na linha mediana para minimizar a taxa de complicações da ferida: ${ }^{13}$

- Usar fio de sutura monofilamentar de absorção lenta ou fio não absorvível.

- Um fio de sutura $n^{\circ} 2 / 0$ é suficiente - montado em agulha cortante 
Intervenções fundamentais em cirurgia: diérese, hemostasia e síntese Medeiros AC, Dantas-Filho AM

- Usar sutura contínua ancorada

- Fechar a ferida englobando apenas a aponeurose, evitando incluir o músculo

- Evitar sutura sob tensão

- Afrontar mas não comprimir excessivamente as bordas da ferida

- Pontos somente na aponeurose, de 5 a $8 \mathrm{~mm}$ da borda da ferida

- Usar intervalos de 5 a $8 \mathrm{~mm}$ de distância entre os pontos

Para auxiliar nas suturas são utilizados mais frequentemente os porta-agulhas de Mayo-Hegar e de Mathieu e pinças anatômicas sem dentes e com dentes de rato. (Figuras 10 e 11). A síntese dos ferimentos pode ser realizada com pontos simples separados ou com sutura contínua. Na pele, o ponto simples, a sutura contínua e a intradérmica são estéticas. O ponto de Donatti é hemostático, mas apresenta resultado estético de má qualidade, pois o trauma à pele é maior do que no uso do ponto simples. Assim, deve ser empregado nos ferimentos do couro cabeludo, região perineal e feridas complexas. Os músculos devem ser aproximados sem tensão, a fim de evitar a isquemia e o esgarçamento, de preferência com pontos em U.

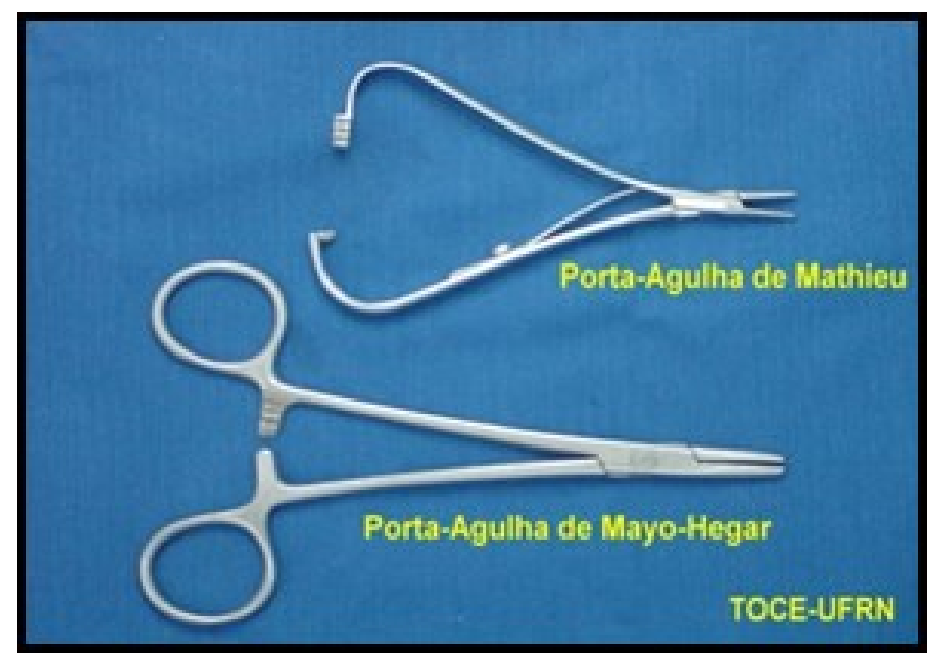

Figura 10 - Porta-agulhas de Mathieu e de Mayo-Hegar. 


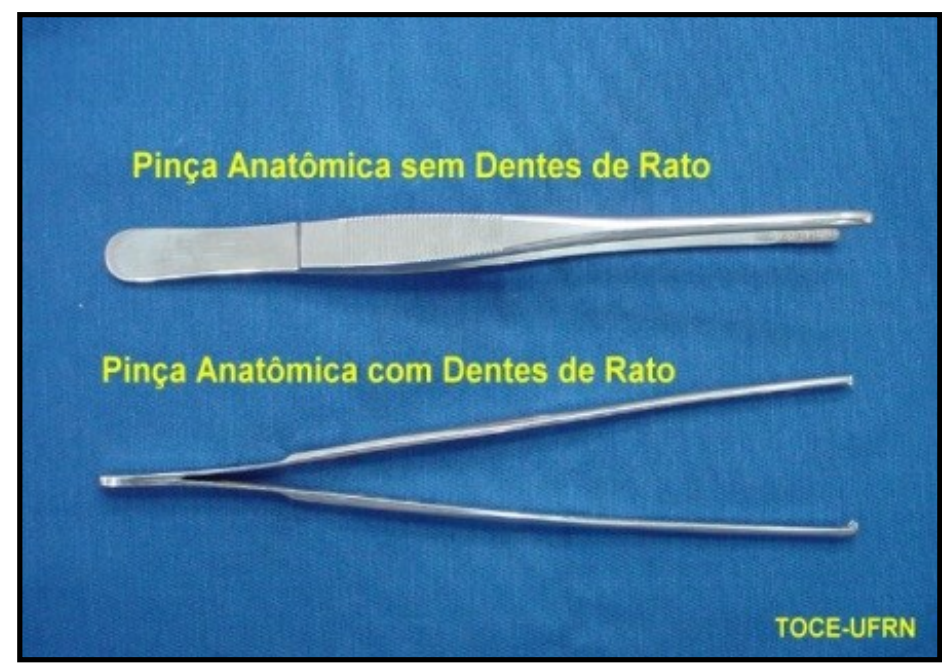

Figura 11 - Pinças anatômicas

\section{CONDIÇÕES DE UMA BOA SÍNTESE}

$\mathrm{Na}$ execução das manobras de síntese tem-se que observar alguns princípios, que são fundamentais para obter uma boa e rápida cicatrização da ferida, sem infecção.

1. Assepsia e antissepsia local - Na ausência destas poder-se-á obter um bom afrontamento imediato, que desaparecerá logo que sobrevenha a infecção.

2. Bordas nítidas - A importância reside no fato de que a irregularidade das bordas condiciona má coaptação e cicatriz defeituosa.

3. Hemostasia - Graças a uma hemostasia perfeita, evita-se qualquer sangramento que, infiltrando ou separando as bordas da ferida, determinará a formação de hematoma e infecção.

4. Afrontamento anatômico - As suturas devem ser feitas plano por plano, sem interposição de corpos estranhos, sem espaços mortos, que dariam lugar a coaptação defeituosa dos tecidos, favorecendo a formação de seromas, deiscência e infecção.

5. Tração moderada - As bordas das feridas devem ser suturadas sem pressão exagerada, evitando transtorno à irrigação sanguínea, isquemia e necrose de tecidos.

6. Material adequado - Deve-se usar fio de sutura com todas as qualidades do fio ideal, dotado de agulha adequada aos órgãos e tecidos a serem suturados.

7. Boa técnica - Deve ser delicada, com passagem suave da agulha, obedecendo a sua curvatura, abrangendo em cada passagem a menor quantidade possível de tecido, com segurança. 
Intervenções fundamentais em cirurgia: diérese, hemostasia e síntese

Medeiros AC, Dantas-Filho AM

\section{MÉTODOS ALTERNATIVOS DE SÍNTESE}

\section{Sutura mecânica}

A utilização dos grampeadores, aplicados de forma adequada, aproxima eficientemente as bordas da ferida de forma rápida, e com resposta inflamatória previsível. Isto reduz a incidência de infecção nas feridas contaminadas e potencialmente contaminadas. A maior desvantagem da sutura mecânica é o alto custo ${ }^{17}$. Alguns estudos têm demonstrado complicações acima de 50\%, comparando com as suturas manuais ${ }^{18,19}$.

\section{Fitas adesivas}

A utilização de fitas adesivas é um método simples para aproximação das feridas superficiais sem tensão. A fita mais empregada é a de poliuretano microporoso, combinado com o adesivo de polialquilacrato (micropore ${ }^{\circledR}$ ). A porosidade da fita impede o acúmulo de líquido e secreções, o que evita a colonização bacteriana e promove a cicatrização com bons efeitos estéticos ${ }^{20}$. Para receber a fita adesiva a pele deve estar seca. De uma maneira simples, a pele pode ser lavada com solução salina $0,9 \%$ e enxugada/seca com compressa estéril. A fita adesiva pode ser empregada para proteger suturas intradérmicas ou após a retirada precoce dos pontos, com o objetivo de obter bons resultados estéticos.

\section{Adesivo Tópico}

$\mathrm{O}$ adesivo tecidual a base de octil-cianoacrilato (Dermabond ${ }^{\circledR}$ ) é um método eficiente para o reparo de feridas pequenas, principalmente em crianças. A aplicação é rápida, acompanha-se de desconforto mínimo, não demanda retirada de pontos e produz bons resultados estéticos ${ }^{21}$. As bordas devem ser aproximadas manualmente ou com pinças; em seguida empregam-se duas ou três camadas suaves de uma película delgada do líquido sobre a ferida. $O$ adesivo está contra-indicado em feridas com infecção ativa e gangrena, em superfícies mucosas, em áreas de tensão cutânea elevada e em pacientes com hipersensibilidade ao cianoacrilato.

\section{REFERÊNCIAS}

1. Chrysos E, Athanasakis E, Antonakakis S, Xynos E, Zoras O. A prospective study comparing diathermy and scalpel incisions in tension-free inguinal hernioplasty. Am Surg. 2005; 71(4):326-9.

2. Rappaport WD, Hunter GC, Allen R, Lick S, Halldorsson A, Chvapil T, Holcomb M, Chvapil M. Effect of electrocautery on wound healing in midline laparotomy incisions. Am J Surg. 1990;160(6):618-20. 
Intervenções fundamentais em cirurgia: diérese, hemostasia e síntese Medeiros AC, Dantas-Filho AM

3. Kearns SR, Connolly EM, McNally S, McNamara DA, Deasy J. Randomized clinical trial of diathermy versus scalpel incision in elective midline laparotomy. Br J Surg. 2001;88(4):41-4.

4. Ji G, Wu Y, Wang $X$, et al. Influence of high-frequency electric surgical knife on healing of abdominal incision, experimental and clinical studies. World J Gastroenterol. 2006;12:4082-5.

5. Garcia A, Nascimento JEA, Darold EM, et al. Healing of abdominal wall aponeurosis of rats after incision with either cold scalpel or electrocautery. Acta Cir Bras. 2007:22(Suppl.1):12-5.

6. Huang J, $Y u$ Y, Wei $C$, Qin $Q$, Mo $Q$, Yang W. Harmonic scalpel versus electrocautery dissection in modified radical mastectomy for breast cancer: A meta-analysis. PLoS One. 2015 Nov 6;10(11):e0142271.

7. Guarini D, Gracia B, Ramírez-Lobos V, Noguera-Pantoja A, Solé-Ventura P. Laser biophotomodulation in patients with neurosensory disturbance of the inferior alveolar nerve after sagittal split ramus osteotomy: A 2-Year follow-up study. Photomed Laser Surg. 2018 ;36(1):3-9.

8. Hu HC, Lin SY, Hung YT, Chang SY. Feasibility and associated limitations of officebased laryngeal surgery using carbon dioxide lasers. JAMA Otolaryngol Head Neck Surg. 2017;143(5):485-91.

9. Figurová $M$, Ledecký V, Karasová M, Hluchý $M$, Trbolová $A$, Capík I, Horňák $S$, Reichel P, Bjordal JM, Gál P. Histological assessment of a combined low-level laser/light-emitting diode therapy $(685 \mathrm{~nm} / 470 \mathrm{~nm})$ for sutured skin incisions in a porcine model: A short report. Photomed Laser Surg. 2016;34(2):53-5.

10. Goldenberg S, Bevilacqua RG. Bases da Cirurgia. Edusp, São Paulo, 1981.

11. Wang M, Tan H, Wu Z, Liang Y. The efficacy and safety of anti-fibrinolytic agents in blood management following peri-acetabular osteotomy: A meta-analysis. Medicine (Baltimore). 2018;97(34):e11967.

12. Novotny R, Hlubocký J, Mitáš $P$, Lindner J. Fibrin sealants in cardiac surgery: The last five years of their development and application. Adv Clin Exp Med. 2018;27(6):857-62.

13. Israelsson LA, Millbourn D. Closing midline abdominal incisions. Langenbecks Arch Surg. 2012;397(8):1201-7.

14. de Lissovoy G, Fraeman K, Hutchins V, Murphy D, Song D, Vaughn BB. Surgical site infection: incidence and impact on hospital utilization and treatment costs. Am J Infect Control. 2009;37(5):387-97.

15. Israelsson LA, Wimo A. Cost minimisation analysis of change in closure technique of midline incisions. Eur J Surg. 2000;166 (8):642-6.

16. Poulose BK, Shelton J, Phillips S, Moore D, Nealon W, Penson D, Beck W, Holzman MD. Epidemiology and cost of ventral hernia repair: making the case for hernia research. Hernia 2012;16 (2):179-83. 
17. Wehrman WE, Tangren CM, Inge TH. Cost analysis of ligature versus stapling techniques of laparoscopic appendectomy in children. J Laparoendosc Adv Surg Tech A. 2007;17(3):371-4.

18. Farrah JP, Lauer CW, Bray MS, McCartt JM, Chang MC, Meredith JW, Miller PR, Mowery NT. Stapled versus hand-sewn anastomoses in emergency general surgery: a retrospective review of outcomes in a unique patient population. J Trauma Acute Care Surg. 2013;74(5):1187-92.

19. Mackeen AD, Khalifeh A, Fleisher J, Vogell A, Han C, Sendecki J, Pettker C,Leiby BE, Baxter JK, Sfakianaki A, Berghella V; CROSS Consortium. Suture compared with staple skin closure after cesarean delivery: a randomized controlled trial. Obstet Gynecol. 2014;123(6):1169-75.

20. Gkegkes ID, Mavros MN, Alexiou VG, Peppas G, Athanasiou S, Falagas ME. Adhesive strips for the closure of surgical incisional sites: a systematic review and meta-analysis. Surg Innov. 2012;19(2):145-55.

21. Harmon AM, Kong W, Buensuceso CS, Gorman AJ, Muench TR. Effects of fibrin pad hemostat on the wound healing process in vivo and in vitro. Biomaterials. 2011;32(36):9594-601.

22. https://amcgmx.wordpress.com/2012/04/02/el-bisutri-armonico-causanecrosis-mas-profunda-de-tejidos-blandos-comparado-con-el-electrocauteriomonopolara-un-nivel-estandar-de-potencia-en-un-modelo-porcino/. Acesso em 10 de setembro de 2018. 